\title{
A Contribution to the Diagnosis of Capillaria hepatica Infection by Indirect Immunofluorescence Test
}

\author{
Bárbara CA Assis, Liliane M Cunha, Ana Paula Baptista, Zilton A Andrade ${ }^{+}$
}

Centro de Pesquisas Gonçalo Moniz-Fiocruz, Rua Valdemar Falcão 121, 40295-001 Salvador, BA, Brasil

A highly specific pattern of immunofluorescence was noted when sera from Capillaria hepatica-infected rats were tested against the homologous worms and eggs present either in paraffin or cryostat sections from mouse liver. The pattern was represented by a combined apple green fluorescence of the internal contents of worms and eggs, which persisted in serum-dilutions of 1:400 up to 1:1600. Unequivocal fluorescent pattern was observed from 15 days up to 3 months following inoculation of rats with embryonated $\mathrm{C}$. hepatica eggs and such result was confirmed by the ELISA. After the 4th month of infection, the indirect immunofluorescence test turned negative, probably revealing the extinction of parasitism, however the ELISA was contradictory, disclosing high levels of antibodies in this period. The IIF was also negative when control normal rat sera and sera from rats administered by gavage with immature C. hepatica eggs (spurious infection), or for reactions made against Schistosoma mansoni eggs, although a weakly positive pattern occurred with Fasciola hepatica eggs. The indirect immunofluorescence test may be recommended for use with human sera to detect early $\mathrm{C}$. hepatica infection in special clinical instances and in epidemiological surveys, since it is a simple, inexpensive, and reliable test, presenting excellent sensitivity and specificity. Although the diagnosis is positive only during early infection, this is the period when the symptoms are usually more severe and the need for differential diagnosis is greater.

Key words: Capillaria hepatica - immunofluorescence test - rat capillariasis

Capillaria hepatica is a nematode of worldwide distribution, especially frequent in rodents and lagomorphis, but that can also parasitize man (Cochrane et al. 1957, Piazza et al. 1963, Cislaghi \& Radice 1970, Berger et al. 1990, Choe et al. 1993). Natural infection of rats in the large cities runs about $60 \%$ or more (Luttermoser 1938, Wright 1961, Galvão 1976, Chieffi et al. 1981, Juncker-Voss et al. 2000). Transmission to man may also be frequent where precarious hygiene prevails, especially in children. There are about 40 human cases published in the world literature, the great majority being autopsy reports from instances of extremely severe infections (Cochrane et al. 1957, Piazza et al. 1963). It has been suspected that less severe cases usually pass unnoticed (Galvão 1979, 1981, Bhattacharya et al. 1999, Juncker-Voss et al. 2000). There are plenty of reasons for that. First, the clinicians rarely think of $C$. hepatica as a parasite of man, since the current literature leaves the impression that hepatic capillariasis is an exceptionally rare infection of man. Also, clinical manifestations in milder cases probably share signs and symptoms common to many other trivial conditions. However, the main hindrance to diagnosis is related to the life cycle of the parasite. No eggs appear in the host feces. Eggs are retained in the carcass of infected ani-

Financial support: Papes III

${ }^{+}$Corresponding author. Fax: +55-71-365-2155.

E-mail: zilton@cpqgm.fiocruz.br

Received 9 July 2003

Accepted 23 January 2004 mals, but still need to rest for some 28-30 days in the environment, exposed to adequate conditions of moisture and temperature, in order to become infecting. Adult $C$. hepatica worms lives a transient life inside the host liver. Soon after egg lying the worms die and disintegrate, causing focal necro-inflammatory lesions. These lesions, containing worm debris and eggs, tend to heal by encapsulation, calcification, and resorption (Luttermoser 1938, Ferreira \& Andrade 1993, Gotardo et al. 2000). It can be presumed that during the formation of such hepatic lesions some degree of painful liver enlargement, accompanied by fever, splenomegaly, and peripheral eosinophilia may follow, which are probably undistinguishable from other "tropical eosinophilia" syndromes (Beaver et al. 1952, Beaver 1969, Kaplan et al. 2001). The possibility to detect these cases depends on our awareness about the role $C$. hepatica may play in human pathology, and the availability of a good immunological test. Indirect immunofluorescence test (IIF), made on histological slides containing parasite and eggs, have been tried with good results. Such test have been considered to be practical, reliable and sensible (Galvão 1976, 1981, Juncker-Voss et al. 2000). The present study confirms and extends these previous results by experimentally testing the sensibility and specificity of the method, and by investigating the influence of several factors on its performance, such as time and type of infection, parasite load, cross reactivity, serum dilution, and type of histological material utilized.

\section{MATERIALS AND METHODS}

Infection and experimental groups - Thirty-five Wistar adult rats, males and females, kept on a balanced commercial diet and water ad libitum, were infected by the administration of embrionated $C$. hepatica eggs, by means of a gastric tube. The eggs had been collected from the livers 
of rats or mice by the 40-50 days after experimental infection, as described elsewhere (Gotardo et al. 2000, Souza et al. 2001). The animals were divided into four groups, as follows:

Group 1 - Ten rats were infected with 600 embryonated eggs, representing a true infection with high worm load. The inoculum was chosen in order to produce massive infection, according to Oliveira and Andrade's data (2001).

Group 2 - Ten rats infected with 30 embryonated eggs, representing a true infection with low worm load.

Group 3 - Ten rats were administered with 600 immature eggs, representing a so-called spurious infection (Nascimento \& Sadigursky 1986).

Group 4 - Five rats were administered with saline by a gastric tube, representing normal non-infected controls.

Sera tested - The sera used for the IIF and ELISA were collected from the tail vein of Wistar rats from the four groups. About $0.5 \mathrm{ml}$ of blood was drawn at a time. After coagulation, the blood was centrifuged at $14,000 \mathrm{rpm} / 3 \mathrm{~min}$, and the serum collected and stored at $-20^{\circ} \mathrm{C}$ until the moment of use. The sera were collected at the following experimental points: $0,9,15,18$, and 40 days, and 4,7 , and 12 months following infection of the animals.

$I I F$ - All the tests were made on histological sections taken from the livers of CBA mice on the 3rd-4th week after infection with 150 embryonated $C$. hepatica eggs. This is a heavy infection, well tolerated by mice. Fragments from these livers were either fixed in neutral $10 \%$ formalin and embedded in paraffin or mounted in Tissuetek (Sakura, Tokyo, Japan) and immediately frozen in liquid nitrogen and cut in a cryostat at $-20^{\circ} \mathrm{C}$. Indirect immunofluorescence test was used to detect the presence of $C$. hepatica antigens in the sections of mouse liver. Sections were either used with previous treatment for antigen recovering or not. In the first case the parafinized sections were place in $\mathrm{pH} 6$ citrate buffer and subjected to three cycles of micro-wave treatment. Sections were covered with sera (primary antibodies), diluted in phosphate buffered saline, $\mathrm{pH} 7.2$, during $30 \mathrm{~min}$ at $37^{\circ} \mathrm{C}$. Sera dilutions were 1:50, 1:100, 1:200, 1:400, 1:800 and 1:1600. These same sera were tested against sections of mouse liver containing either S. mansoni or F. hepatica eggs. PBS replaced the sera in negative control slides. After a series of washing with iced buffered saline, sections were treated with anti-rat IgG conjugated to fluorescein-isothiocyanate (Sigma, Saint Louis, MO) under a 1:640 dilution. Slides were mounted in carbonate buffer and examined with a Zeiss microscope under UV light, with appropriate barrier and exciter filters.

ELISA - Sera were submitted to an ELISA test for detection of total antibodies, by using a goat anti-rat $\operatorname{IgG}$ conjugated to peroxidase at 1:1,000.(Sigma). The plates were sensitized with $10 \mu \mathrm{g} / \mathrm{ml} /$ per well of $C$. hepatica-egg and worm antigen, diluted in carbonate buffer, $\mathrm{pH} 9.9$. Sera were diluted 1:1000 in PBS with 3\% skimmed milk- and $0.05 \%$ Sigma tween-20. All tests were used in duplicates, including the blank. Lecture was made on a microplate reader "Molecular devices-Thermomax" spectrophotometer (Sunyvaley, CA) under $450 \mathrm{~nm}$ wave-length, connected to a computer with MDS-Soft Max.

\section{RESULTS}

The sections taken from the livers of mice experimentally infected with $C$. hepatica contained well-preserved worms, their eggs and disintegrating worms. The details about the fluorescence staining were centered on the sections of well-preserved worms and eggs. When treated with saline and examined under UV light, only a yellowish auto-fluorescence was observed in the egg shell. Sera from normal intact rats and from those with spurious infection gave essentially negative results. However, sera with 1:50 up to 1:200 dilutions exhibited non-specific fluorescence (Fig. 1). This appeared at the worm cuticle in the egg-shell. The yellowish auto-fluorescence of the eggshell then acquired a bright whitish hue, while its inner contents appeared uniformly black.

When treated with sera from $C$. hepatica-infected rats, the structures of worms and eggs presented a strong applegreen fluorescence, mainly marked at the internal genitalia (uterus) and the hypodermis (especially at the so-called "bacillary bands") (Fig. 2). The eggs were strongly marked at the shell, and their interior appeared empty most of times. However, when the contents of the eggs were preserved in the sections, they were strongly fluorescent. This staining of eggs and worms represented a highly specific feature, present in positive cases even at the highest dilutions, and entirely absent from controls. Actually, nine days after inoculation the results were still negative, but turned weakly positive by the 15th day (Table) even at a 1:1600 dilution. However, the 15-day positive result was only observed in slides submitted to antigen retrieval. From then on, a strong positive reaction was regularly observed during the first three months following infection, with or without the retrieval of antigen. The positive fluorescence and its patterns occurred in infected rats regardless of parasite load. However, with serum dilutions of 1:400 or higher, it did not persist when $C$. hepatica infection had lasted for 4 months or over. Sera from rats with 4,7 or 12 month-old infection revealed consistent negative results by IIF, although anti-C. hepatica antibodies continued to be detected by ELISA (Fig. 3). When the IIF was made using rat anti-C. hepatica serum, against tissue containing Schistosoma mansoni eggs, results were negative. The same test using Fasciola hepatica eggs resulted in a weakly positive reaction even when dilutions of 1:400 and over were used.

\section{DISCUSSION}

Examining 500 children from low income families in Salvador, BA, Brazil, Galvão (1979) found that $44.2 \%$ of them exhibited several patterns of positive fluorescence for $C$. hepatica in the sera eluted from a drop of blood collected in filter paper. In nine cases fluorescence was particularly strong, disclosing a peculiar pattern even under high dilutions. This pattern was characterized by the fluorescence of the inner contents of the eggs and of the internal structures of the worms, particularly at the gonads. The present study confirms these results and adds a further detail, the focal staining of "bacillary bands", which are present just below the worm cuticle. These combined fluorescent sites formed a highly characteristic pat- 

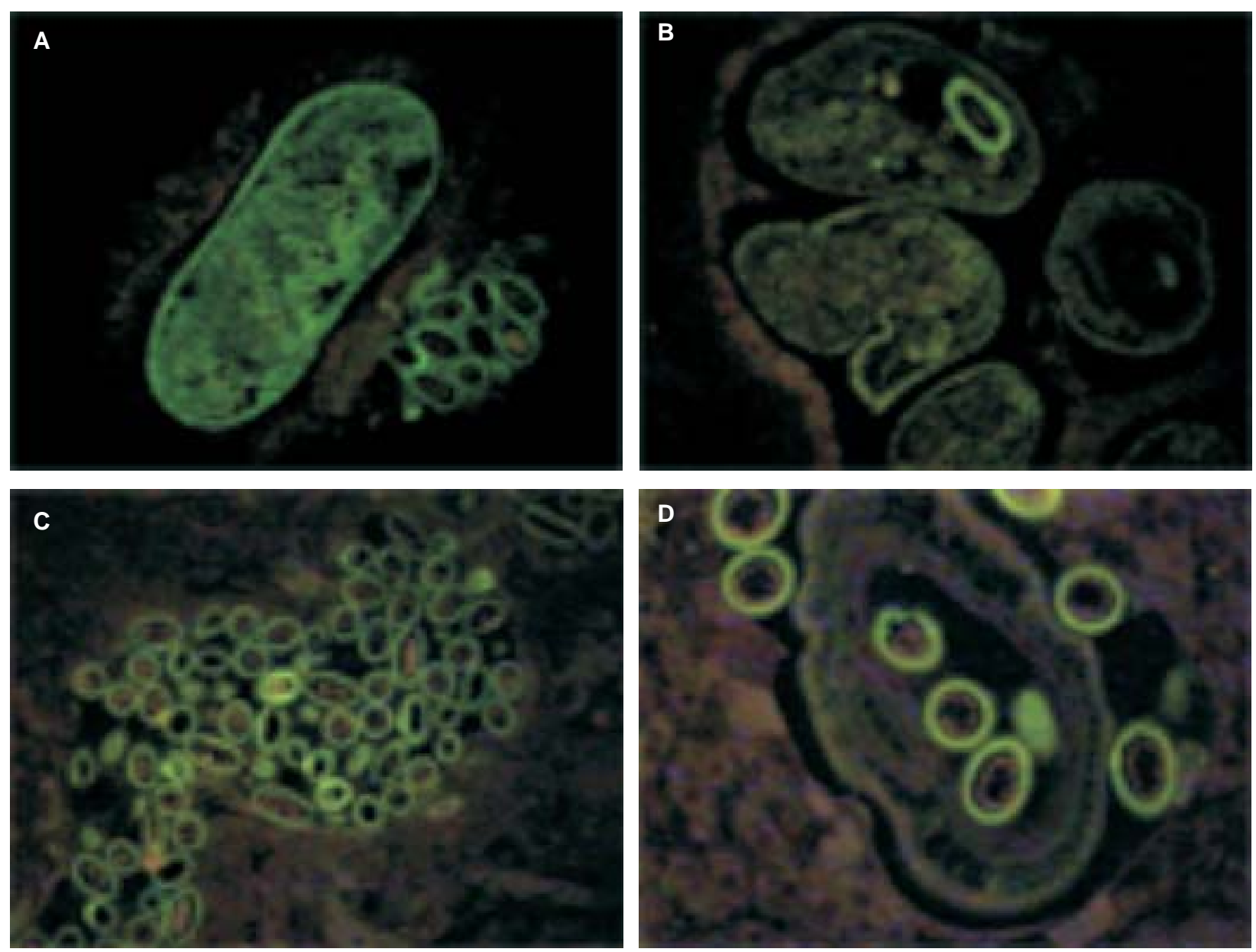

Fig.1: sections of mouse liver, containing Capillaria hepatica worms and eggs, treated with control serum from normal rats. A: diffuse fluorescence revealing the internal structures of the worms, especially the digestive tube and also the cuticle. The egg shells exhibit an apple green fluorescence. Intensity of fluorescence progressively diminishes with increasing in dilutions, as can be noted by comparing the picture at A (1:50) with that at B (1:100); C: collections of eggs treated with 1:400 serum dilution. The internal contents of the eggs lack fluorescence. The worm seen at D is also unstained. Magnification 200X, except D, 400X.

tern disclosed by the positive sera (Fig. 1). The internal structures of the worms appeared marked by the serum taken at the 15th day of infection, but the internal contents of the eggs appeared a little later, around the 18th day. From then on, the typical pattern persisted unchanged throughout the first three months of infection. Therefore, the positive fluorescent pattern appeared early in the course of $C$. hepatica infection, was not influenced by the worm load, by was so by the presence of viable eggs within the host tissue. By the 4th month after inoculation, fibrosis decreased and the eggs probably became metabolically inactive. Luttermoser (1938) observed a gradual reduction of the viability of the eggs with the increasing age of the infections. A trial to embryonate eggs after 12 months of infection resulted unsuccessful (data not shown). Apparently the negative finding observed in old infection was not related to the level of ELISA antibodies, but to their quality. As a matter of fact, sera taken 15 days after infection revealed a definite pattern of positive fluorescence, while presenting ELISA-levels of antibodies lower than those from animals with old infections (four months or more). Therefore, results from anti-C.hepatica antibodies searched by the ELISA method in infected rat sera were concordant with those revealed by the IIF only during the first three months after infection. From then on a diverging result was noted. Besides, positive fluorescence was never observed in control sections or in cases of $C$. hepatica spurius infection (animals subjected to ingestion of non-infective immature eggs). Spurious infection has been claimed to sensitize the host on the basis of the ELISA test (Nascimento \& Sadigursky 1986), a finding not confirmed in the present investigation, especially when the IIF was applied. Fluorescence was then weak and non-specific, disappearing when dilutions of 1:400 or over were employed.

Although the tests made to investigate cross reactivity with other helmints were very preliminary in the present study, revealing a weakly positive result with $F$. hepatica eggs and totally negative results with $S$. mansoni eggs, there are suggestions in the literature that such cross 

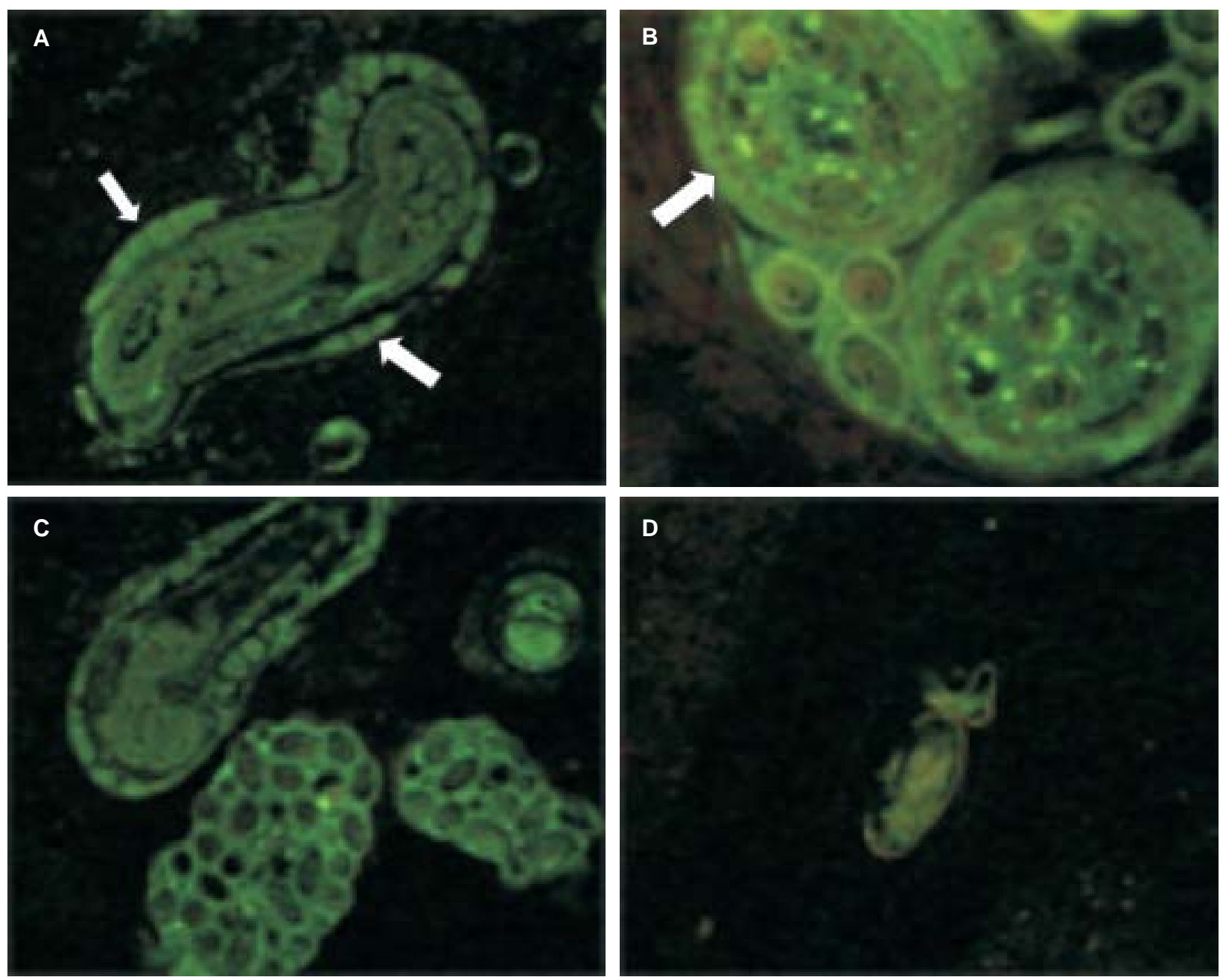

Fig. 2: sections of mouse liver, containing Capillaria hepatica worms and eggs, treated with sera from rats with 18-day old C. hepatica infection. Bacillary bands (A, arrows) appear fluorescent, as well as the uterus (B, arrow) and the internal contents of the eggs (C). Serum dilutions: A 1:1,600 and B 1:800; C 1:2,600. Picture at the D represents fluorescence of Fasciola hepatica egg after treatment with $C$. hepatica rat serum, dilueted at 1:800. Magnifications: B 400X; all others 200X.

TABLE

Results of the indirect immunofluorescence test according to intensity and patterns and in relation to variations in serum dilutions

\begin{tabular}{lcccc} 
Serum dilution & Normal controls & Heavy true infection ${ }^{a}$ & Mild true infection ${ }^{a}$ & Spurius infection \\
\hline $1: 50$ & + & +++ & +++ & + \\
$1: 100$ & + & +++ & +++ & + \\
$1: 200$ & + & +++ & +++ & + \\
$1: 400$ & - & +++ & +++ & - \\
$1: 800$ & - & +++ & +++ & - \\
$1: 1600$ & - & ++ & ++ & - \\
\hline
\end{tabular}

$a$ : sera taken 15-40 days after infection; -: negative; +: mild and non-specific; ++: moderate and specific; +++: strong and specific

reactivity is not to be expected, at least when high serum dilutions are used. Galvão (1979) observed the C. hepatica IIF to be negative in several instances when the sera of children presenting eggs of intestinal helminths in stools were examined. Junker-Voss et al (2000) obtained negative results with sera from cases of Echinococcus granulosus, E. multilocularis, F. hepatica. S. mansoni, Toxo- cara canis, Trichuris trichiura, and Trichinella spiralis infections. Of course the possibility of cross reactivity has not been ruled out and should be better explored in further studies. But, one should consider that a good $C$. hepatica immunological test is to be useful for especial clinical situations and epidemiological surveys as strong presumptive evidence, not necessarily as an absolute di- 


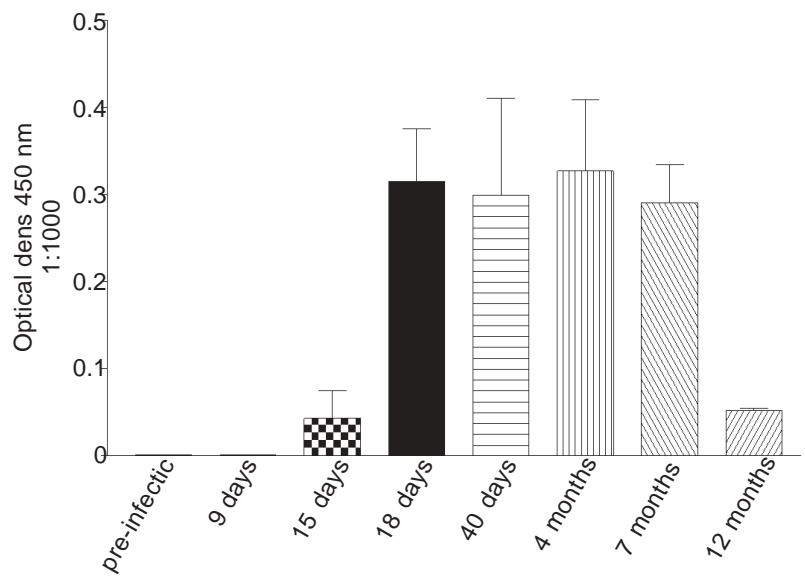

Fig. 3: levels of anti-Capillaria hepatica antibodies in the sera of infected rats in relation to the days following infection (ELISA).

agnosis. In special cases, complementary data should be sought through clinical investigation, sometimes including such procedures as ultrasound imaging and liver biopsy.

Examinations made on paraffin sections allow the same results as when cryostat sections were used. As a matter of fact, paraffin sections yielded better localization and definition of fluorescent areas. The practical advantages of paraffin over cryostat sections are obvious. It is easier to keep paraffin blocks and unstained sections for future investigations, and at lower cost, than frozen tissue and sections. Micro-wave antigen enhancement yields brighter and crisp positive fluorescence, but its absence will not interfere with any other aspects of the test.

Considering the high prevalence of $C$. hepatica infection of rats in large cities and the poor hygienic conditions sometimes prevailing in the poor human populations at their outskirts, it seems logical to suspect that human infections with this helminth can occur in such environment. A good serological test for the diagnosis of $C$. hepatica infection should be included for the differential diagnosis when children or even adults present hepato-splenomegaly, unexplained fever and eosinophilia. It is hoped that the availability of a reliable and practical diagnostic tool will facilitate future investigations to determine the real role $C$. hepatica infection plays in human pathology.

\section{REFERENCES}

Beaver PC 1969. The nature of visceral larva migrans. J Parasitol 55: 3-12.

Beaver PC, Snyder CH, Carrera GM, Dent JH, Lafferty JW 1952. Chronic eosinophilia due to visceral larva migrans. Report of three cases. Pediatrics 9: 7-19.
Berger T, Degrémont A, Gebbers JO, Tonz O 1990. Hepatic capillariasis in a 1-year-old child. Eur J Pediatr 149: 333336.

Bhattacharya D, Patel AK, Das SC, Sikdar A 1999. Capillaria hepatica, a parasite of zoonotic importance $=$ A brief overview. J Commun Dis 31: 267-269.

Chieffi PP, Dias RM, Mangini AC, Grispino DM, Pacheco MA 1981. Capillaria hepatica (Bancroft 1893) in Muridae trapped in the municipality of São Paulo, Brazil. Rev Inst Med Trop São Paulo 23: 143-146.

Choe G, Lee HS, Seo JK, Chai JY, Lee SH, Eom KS, Chi JG 1993. Hepatic capillariasis: first case reported in the Republic of Korea. Am J Trop Med Hyg 48: 610-625.

Cislaghi F, Radice C 1970. Infection by Capillaria hepatica. First case report in Italy. Helv Paediatr Acta 25: 647-654.

Cochrane JC, Sagorin L, Wilcocks MG 1957. Capillaria hepatica infection in man. A syndrome of extreme eosinophilia, hepatomegaly and hyperglobulinaemia. S AfrMed J 31: 751-755.

Ferreira LA, Andrade ZA 1993. Capillaria hepatica: a cause of septal fibrosis of the liver. Mem Inst Oswaldo Cruz 88: 441-447.

Galvão VA 1976. Capillaria hepatica, estudo da incidência em ratos de Salvador, Bahia, e dados imunopatológicos preliminares. Rev Soc Bras Med Trop São Paulo 10: 333-337.

Galvão VA 1979.Tentativa para detectar infecção por Capillaria hepatica no homem. Rev Inst Med Trop São Paulo 21: 231-236.

Galvão VA 1981. Capillaria hepatica: an evaluation of its pathogenic role in man. Mem Inst Oswaldo Cruz 76: 415-433.

Gotardo BM, Andrade RG, Andrade ZA 2000. Hepatic pathology in Capillaria hepatica infected mice. Rev Soc Bras Med Trop 34: 341-346.

Juncker-Voss M, Prosl H, Lussy H, Enzenberg U, Auer H, Nowotny N 2000. Serological detection of Capillaria hepatica by indirect immunofluorescence assay. J Clin Microbiol 38: 431-433.

Kaplan KJ, Goodman ZD, Ishak G 2001. Eosinophilic granuloma of the liver. A characteristic lesion with relationship to visceral larva migrans. Am J Surg Pathol 25: 1316-1321.

Luttermoser GW 1938. An experimental study of Capillaria hepatica in the rat and the mouse. Am J Trop Med Hyg 27: 321-340.

Nascimento I, Sadigursky M 1986. Capillaria hepatica: alguns aspectos imunopatológicos da infecção espúria e da infecção verdadeira. Rev Soc Bras Med Trop 19: 21-25.

Oliveira RF, Andrade ZA 2001. Worm load and septal fibrosis of the liver in Capillaria hepatica-infected rats. Mem Inst Oswaldo Cruz 96: 1001-1003.

Piazza R, Correa MA, Fleury RN 1963. Sobre um caso de infestação humana por Capillaria hepatica. Rev Inst Med Trop São Paulo 5: 37-41.

Souza MM, Paraná R, Trepo C, Barbosa Jr AA, Oliveira I, Andrade ZA 2001. Effect of interferon- $\alpha$ on experimental septal fibrosis of the liver. Study with a new model. Mem Inst Oswaldo Cruz 96: 343-348.

Wright KA 1961. Observations on the life cycle of Capillaria hepatica (Bancroft, 1893) with a description of the adult. Can J Zool 38: 167-182. 